\title{
Bases épidémiocliniques de la maladie du Krafft chez le dromadaire (Camelus dromedarius) dans le Sud tunisien
}

\author{
M. Seddik Mabrouk ${ }^{1 *}$ T. Khorchani ${ }^{1}$ \\ M. Benromdhane ${ }^{2}$
}

\section{Mots-clés}

Camelus dromedarius - Pâturage Maladie de carence - Carence minérale - Tunisie.

\section{Résumé}

Cette étude présente une observation descriptive, analytique, épidémiologique et clinique de la maladie du Krafft, ainsi qu'une évaluation des risques d'apparition de la maladie par la recherche des facteurs de réceptivité, dans les régions du sud de la Tunisie. Les symptômes du Krafft se caractérisent par des troubles locomoteurs allant de la boiterie à l'immobilisation qui aggrave l'état général de l'animal affecté. La prévalence moyenne de la maladie a été de 5 p. 100 dans les troupeaux étudiés. Elle a été liée au passage des troupeaux vers les parcours de Dhahar. Elle a surtout été observée en hiver et au printemps chez les femelles en fin de gravidité et au début de la lactation.

\section{INTRODUCTION}

En Tunisie, la quasi-totalité du cheptel camelin vit dans les régions arides et désertiques caractérisées par des parcours à faible productivité. Pour satisfaire les besoins alimentaires des animaux, la majorité des éleveurs ont mis en œuvre une stratégie de gestion des parcours basée sur la mobilité des troupeaux. La transhumance entre les différentes régions du sud du pays permet de minimiser les charges financières liées à l'alimentation.

Toutefois, il existe une maladie particulière aux troupeaux du Sud, connue sous le nom de Krafft, qui pèse sur la conduite et la rentabilité de l'élevage. Diverses hypothèses évoquant un déséquilibre alimentaire en excès ou en déficit ont été émises dans les rares

\footnotetext{
1. Laboratoire d'élevage et de la faune sauvage, Institut des régions arides, 4119 Médenine, Tunisie.

2. Ecole nationale de médecine vétérinaire, Sidi-Thabet, Tunisie.

* Auteur pour la correspondance

Tél. : +216756330 05; fax : +21675633006

E-mail : seddik.mouldi@ira.rnrt.tn
}

études antérieures $(1,4,7,8)$. Cette maladie est caractérisée par l'atteinte de l'appareil locomoteur des animaux, entravant leurs déplacements et perturbant le programme de transhumance souhaité par l'éleveur. Le présent travail a eu pour objectif de réaliser une étude épidémiologique de la maladie du Krafft dans les régions du sud-est de la Tunisie, d'évaluer son impact sur l'animal sur le plan clinique et d'identifier les facteurs prédisposants.

\section{MATERIEL ET METHODES}

Une enquête épidémiologique, basée sur l'examen clinique et un questionnaire auprès des éleveurs et des chameliers, a été effectuée par tirage aléatoire sur les principaux parcours du Sud tunisien. Elle a concerné 28 chameliers possédant au total 2660 têtes dont 630 pâturaient les parcours d'El Ouara Benguerdane. Le reste de l'effectif (2 030) pratiquait la transhumance sur les deux autres principaux parcours du Sud-Est : El Ouara Tataouine et Dhahar.

Les sites visités ont été repérés à l'aide d'un système mondial de positionnement (GPS) et les troupeaux à l'aide de jumelles. Il s'agissait des parcours : 
- d'El Ouara Benguerdane dont la superficie est estimée à 164000 ha et appartient au gouvernorat de Médenine ;

- d'El Ouara Tataouine, situé en continuation du précédent, et dont la superficie est de 400000 ha ;

- de Dhahar, plateau séparé de la plaine de Jaffera par la chaîne montagneuse de Matmata, limité à l'ouest par le grand erg oriental, au nord par la ligne reliant Matmata à Bir Soltan, et au sud par la ligne reliant l'erg des M'Razig et Djnaine. Le Dhahar couvre une superficie de 1235500 ha repartis sur quatre gouvernorats : Tataouine, Kebili, Médenine et Gabes (figure 1).

La zone d'étude est caractérisée par deux influences climatiques très différentes : le Sahara côté ouest, au climat subtropical saharien chaud et sec, et le golfe de Gabes côté nord-est, au climat méditerranéen, tempéré chaud et relativement humide. Les températures sont très variables d'une région à l'autre, avec des valeurs moyennes de $22,5,20$ et $38{ }^{\circ} \mathrm{C}$, respectivement en automne, en hiver, au printemps et en été. L'amplitude thermique annuelle moyenne est de 19,6. Les précipitations sont faibles, irrégulières, et interviennent en hiver et au printemps. La moyenne annuelle est de $124 \mathrm{~mm}$ à El Ouara mais ne dépasse pas $50 \mathrm{~mm}$ dans les zones les plus sahariennes.

\section{RESULTATS ET DISCUSSION}

\section{Résultats épidémiologiques}

Les éleveurs enquêtés connaissaient tous la maladie sous le nom de Hchem ou Krafft. Toutefois, certains jeunes chameliers d'El Ouara Benguerdane ne connaissaient pas la symptomatologie de la maladie.

\section{Répartition spatiale de la maladie}

L'enquête a porté sur les principaux parcours du Sud tunisien contrairement aux études précédentes réalisées sur les parcours de Jnein et Bir Aouin par Durand et Chkouk (4), et Zahzah (8). La prévalence de la maladie du Krafft a varié d'une région à une autre. La majorité des cas ont été observés sur les parcours d'Ouara Tataouine et de Dhahar avec une prévalence respectivement de 4,5 et 5,6 (tableau I). Le Krafft était présent dans la majorité des troupeaux pâturant la région d'El Ouara Tataouine et de Dhahar où il pouvait affecter 15 p. 100 des animaux d'un troupeau. Toutefois, il a été inexistant sur le parcours d'Ouara Benguerdane.

Cette maladie réapparaît chaque année dans les élevages extensifs avec une fréquence variable selon les saisons, contrairement aux observations de Durand et Chkouk (4) qui rapportent la présence de la maladie toute l'année. Les résultats montrent qu'elle affecte surtout les femelles en hiver et au printemps, période coïncidant avec la fin de la gestation et le début de la lactation. Pendant cet intervalle, la chamelle a un besoin élevé en éléments minéraux, mais il coïncide avec la période de transhumance vers les parcours de Dhahar. Ainsi, la chamelle est confrontée à des conditions

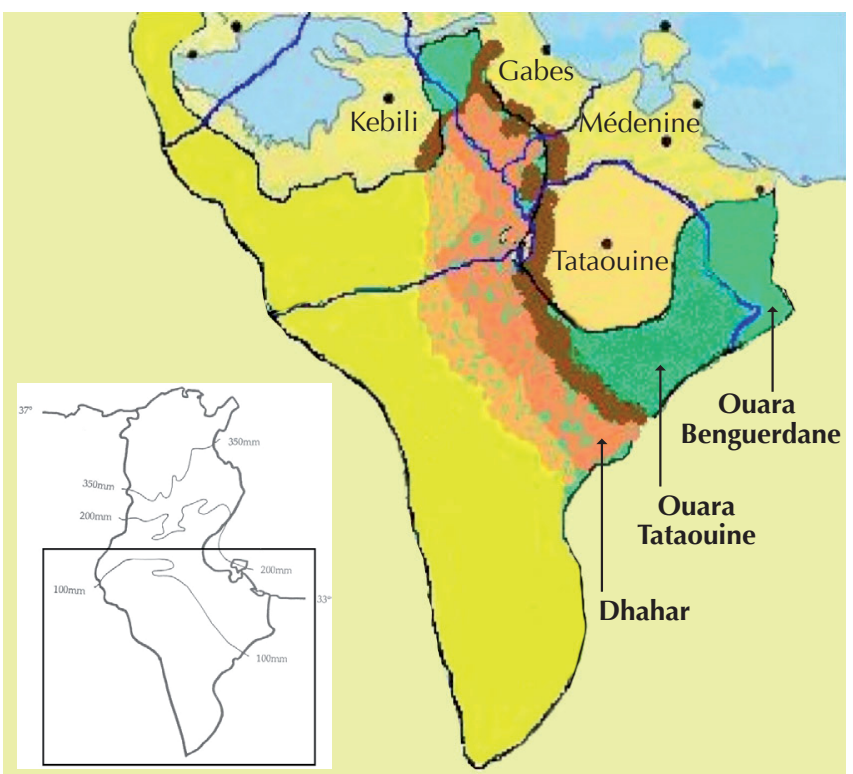

Figure 1 : parcours de dromadaires au sud-est de la Tunisie.

difficiles avec des besoins nutritionnels élevés non compensés par des apports alimentaires adéquats.

\section{Facteurs intrinsèques favorisant l'apparition de la maladie}

- Sexe et état physiologique

D'après les chameliers, le Krafft atteint surtout les femelles. Cette constatation est confirmée par les observations de la présente étude puisque aucun des mâles rencontrés n'a présenté les symptômes de la maladie dans les troupeaux visités. Ceci peut être expliqué par l'augmentation des besoins chez la chamelle pendant la période de début de lactation. Ce facteur n'a pas été étudié par Zahzah (8), toutefois Durand et Chkouk (4) ont décrit la maladie chez les mâles utilisés dans les services militaires sahariens pendant les années 1950. En fait, il semble que cette pathologie soit liée aux périodes physiologiques les plus critiques. En effet, les animaux les plus confrontés à la maladie sont les femelles en fin de gestation et en début de lactation. La majorité des cas rencontrés sont des femelles suitées. Selon les chameliers enquêtés, la maladie a aussi été observée chez les ovins et les caprins utilisant le parcours de Dhahar. Certains chameliers ont même signalé la maladie chez le lièvre dans la région.

- Age

Les adultes ont été les plus touchés par la maladie. La majorité des chameliers ont indiqué que l'âge des animaux malades dépassait souvent deux ans. Seuls, trois d'entre eux ont signalé que la maladie pouvait affecter de jeunes animaux.

\section{Tableau I}

Prévalence de la maladie du Krafft chez le dromadaire selon la région dans le Sud-Est tunisien

$\begin{array}{lccccc}\text { Région } & \text { Nb. de troupeaux } & \text { Nb. d'animaux } & \begin{array}{c}\text { Nb. d'animaux } \\ \text { affectés }\end{array} & \begin{array}{c}\text { Prévalence d'animaux } \\ \text { affectés }\end{array} & \text { Ecart-type } \\ \text { Ouara Tataouine } & 14 & 1300 & 55 & 4,5 & 4,1 \\ \text { Dhahar } & 7 & 580 & 31 & 5,6 & 6,3 \\ \text { Ouara Benguerdane } & 6 & 630 & 1 & 0,1 & 0,2\end{array}$




\section{Facteurs extrinsèques favorisant l'apparition de la maladie}

- Caractéristiques botaniques et pédologiques des parcours

Le parcours de Dhahar est caractérisé par des sols sableux à sableux limoneux. Ces sols sont en majorité développés sur un substrat calcaire plus ou moins superficiel selon les régions. Les horizons sont le plus souvent peu profonds, un affleurement par endroits d'une croute calcaro-gypseuse donnant un aspect caillouteux aux parcours. Ce parcours est couvert par des steppes dominées à l'est par Anthyllis sericea et Rhanterium suaveolens, ainsi que par des espèces xérophiles (Gymnocarpos decander, Helianthemum kahiricum et Artemisia herba alba), et à l'ouest par des espèces psammophiles (Helianthemum confertum, Oudneya africana, Calligonum comosum). Au niveau des dépressions et des zones d'épandage des oueds, les sols sont à voile éolien où dominent les psammophytes comme Retama raetam, Aristida pungens, Calligonum comosum (3). Il est à noter que les dépressions salées sont très rares dans cette région.

Les sols des parcours d'Ouara (Tataouine et Benguerdane) sont de texture sableuse avec une prédominance des dépressions salées. En effet, dans la partie est d'El Ouara (Ouara Benguerdane), caractérisée par une prédominance des dépressions fortement gypseuses et assez salées (5), les plantes halophytes sont plus représentées, surtout Nitraria retusa, Atriplex halimus, Limoniastrum guyonianum, Suaeda mollis et Traganum nudatum. Cependant en allant d'est en ouest (Ouara Tataouine), d'autres espèces, comme Retama raetam, Aristida pungens, Arthrophytum schmittianum, prédominent sur les grandes surfaces de sols profonds (5).

- Mode de gardiennage

Dans toute la zone d'étude, la conduite des troupeaux se fait selon le mode extensif. Le gardiennage des troupeaux dans les zones concernées par l'enquête a lieu de façon continue durant l'année. Néanmoins, certains troupeaux rencontrés sur le parcours de Dhahar étaient sans chameliers et appartenaient à la tribu M'Rezig du gouvernorat de Kebili. En fait, la conduite des troupeaux dans cette région est souvent caractérisée par une période où les animaux sont libres sur parcours sans gardiennage. Cette période est relativement longue et peut atteindre huit mois (de fin février à novembre), empêchant toute intervention ou suivi.

\section{- Mouvements des troupeaux}

L'utilisation des parcours par les dromadaires est différente selon les régions. Les troupeaux des tribus twazine et rbayaâ pâturent les parcours d'El Ouara Benguerdane toute l'année. Le déplacement d'un troupeau à l'intérieur des régions se fait selon le choix du propriétaire et selon l'état du parcours. Le passage de ces troupeaux vers les parcours de Dhahar est très rare.

D'une façon générale, la gestion des espaces pastoraux d'El Ouara Tataouine par les éleveurs dépend des saisons, de l'état des parcours et des besoins hydriques des animaux. La transhumance s'effectue entre les parcours d'El Ouara Tataouine et les parcours de Dhahar. La majorité des chameliers enquêtés passent l'été et l'hiver sur les parcours d'El Ouara Tataouine.

Concernant les parcours de Dhahar, le passage des troupeaux dans cette zone a lieu en automne et au printemps, et est étroitement lié à la pluviométrie. Ainsi, avant tout déplacement au Dhahar, les propriétaires se renseignent sur l'état des différents parcours. A noter toutefois que certains troupeaux pâturent toute l'année dans cette région.

La transhumance des troupeaux vers le parcours de Dhahar a été accompagnée de l'augmentation de la prévalence de la maladie, expliquant l'apparition de celle-ci dans les troupeaux d'Ouara Tataouine (tableau I). Toutefois, dans les troupeaux d'Ouara
Benguerdane, qui sont plus « sédentaires » pour ce parcours, la prévalence de la maladie a été très faible, incriminant les caractéristiques botanique et édaphique du parcours de Dhahar dans l'apparition de la maladie, tout en précisant que sur les deux parcours d'Ouara Tataouine et de Benguerdane il n'y a pas eu de différences dans les pratiques d'élevage de dromadaires suivis par les chameliers (ressources alimentaires et temps de pâturage).

\section{Résultats cliniques}

\section{Symptômes}

La maladie a été suspectée à l'échelle du troupeau par la présence d'un ou de plusieurs animaux qui se sont déplacés lentement et avec difficulté lors du pâturage. L'animal a maigri au fur et à mesure de l'évolution de la maladie, avec une diminution de la taille de la bosse. Certains animaux ont présenté du pica en mâchonnant des os rencontrés sur les parcours ; toutefois, l'appétit est resté normal.

Au début de la maladie, l'animal a présenté une boiterie d'un ou des deux membres antérieurs. Cette boiterie a été nettement perçue le matin lors du départ des animaux au pâturage et a diminué au cours de la journée lorsqu'il faisait plus chaud. Pour chaque troupeau, un lieu de campement a été choisi par le chamelier pour se reposer la nuit. La boiterie est devenue plus visible avec la progression de la maladie, l'animal étant obligé de se reposer plus fréquemment et pouvant se trouver isolé du reste du troupeau. Cette observation n'a pas été rapportée antérieurement. Cette situation serait due à des particularités anatomiques du dromadaire, puisque le poids de l'animal est supporté surtout par les membres antérieurs. La boiterie a évolué par la suite pour s'étendre aux quatre membres. Le dromadaire s'est déplacé alors avec une difficulté extrême. Sa démarche était caractéristique, comparable à celle du caméléon. Le dromadaire s'est immobilisé dans la phase la plus grave de la maladie. Ainsi, le chamelier a rencontré des difficultés pour surveiller ces animaux malades. Les boiteries ont été accompagnées par des craquements articulaires. Elles étaient douloureuses et l'animal a réagi aux moindres manipulations.

L'animal a répugné au baraquage, émettant de forts grognements suite aux douleurs. Il pouvait prendre cette position avec grande difficulté. Toutefois, les chameliers ont signalé que certains animaux malades pouvaient rester toute la nuit en position debout pour éviter les chocs des articulations avec le sol lors du baraquage, tout en émettant des grognements de souffrance. En position baraquée l'animal a essayé de changer de position périodiquement (figure 2).

A l'inspection clinique, aucun traumatisme n'a été observé à l'exception d'une légère déviation des membres antérieurs vers l'extérieur. A la palpation des membres atteints, une légère hypertrophie articulaire a été notée sans être caractéristique des cas examinés. Des nodosités

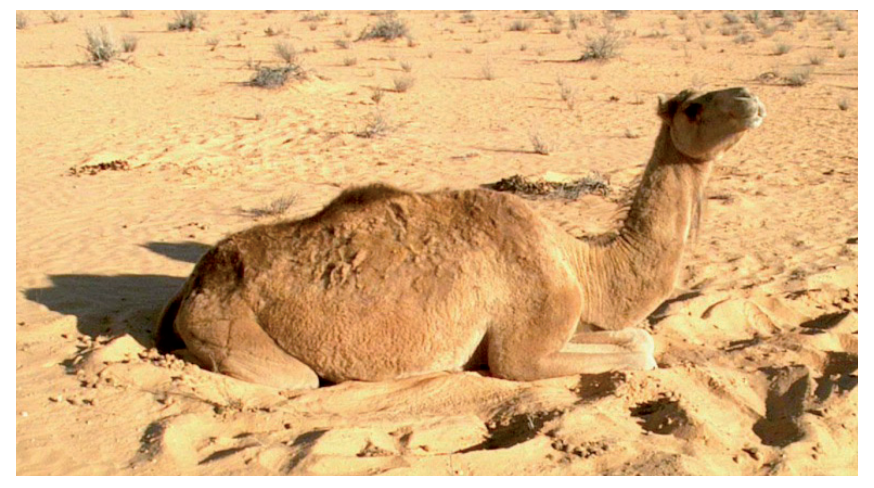

Figure 2 : dromadaire immobilisé par la maladie du Krafft. 
costales ont parfois été présentes, mais ce signe n'a pas été constant non plus. Ces nodosités ont été plus nettes chez des animaux âgés et affectés plusieurs fois par cette maladie. A la palpation/pression des os, l'animal a réagi et exprimé des signes de douleur. La maladie a parfois évolué vers la guérison, surtout si l'éleveur changeait de parcours, ou vers l'aggravation qui a souvent obligé l'éleveur de réformer l'animal malade. Elle a parfois conduit à la mort de l'animal.

\section{Lésions}

L'autopsie d'une chamelle âgée de 16 ans, morte à la suite de la maladie, a montré à l'examen général un état de déshydratation avec présence d'escarres de décubitus. A l'ouverture des deux cavités abdominale et thoracique, la seule lésion observée a été la présence des nodosités costales (figure 3), similaires à celles décrites par Durand et Chkouk (4). Ces nodosités ont été multiples sur les côtes et surtout présentes sur celles du milieu de la cage thoracique ( $3^{\mathrm{e}}, 4^{\mathrm{e}}$ et $5^{\mathrm{e}}$ côtes de chaque côté). Une seule lésion a été observée par côte. Ces lésions, en contact avec le sol lors de la position baraquée du dromadaire, pourraient être dues à des fractures anciennes. En effet, Durand et Chkouk (4) ont émis l'hypothèse d'une fragilisation des os les plus irrigués, siège de mobilisation minérale lors de carences alimentaires, sachant que les os des côtes sont prédisposés à des fractures en raison de la faiblesse de la trame osseuse.

L'examen interne des articulations des carpes et des tarses n'a montré aucune lésion spécifique, malgré une déviation du carpe gauche, observée en position baraquée lors de l'examen clinique ante mortem.

A l'ouverture du rumen, la présence de plusieurs corps étrangers (plastique, petites pierres...) ont indiqué que l'animal avait présenté du pica. Par ailleurs, lors des visites des troupeaux, des signes de pica ont été observés chez les animaux pâturant les parcours de Dhahar et d'El Ouara Tataouine, révélant l'existence de problèmes de carences minérales. Ces signes de pica ont été décrits précédemment par Blajan et Lasnami (2) comme étant une entité pathologique à part. Toutefois, Faye et Bengoumi (6) ont associé le pica chez le dromadaire à une déficience en phosphore.

Les observations cliniques de terrain et les circonstances d'apparition de cette maladie chez le dromadaire sont en faveur d'une déficience d'apport minéral comme étiologie. En effet, chez les autres ruminants, en l'occurrence les bovins, les carences phosphocalciques ont des conséquences pathologiques, marquées chez les

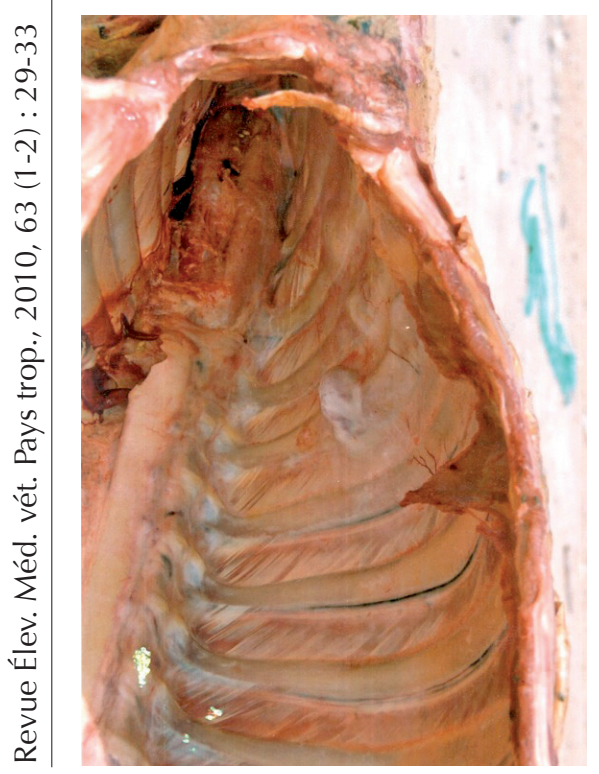

Figure 3 : lésion nodulaire sur les faces internes des côtes d'une chamelle morte suite à la maladie du Krafft. adultes par une déminéralisation généralisée dans les cas d'ostéomalacie, par la décalcification des os et l'augmentation de l'incidence des fractures dans les cas d'ostéoporose, ainsi que par des troubles locomoteurs liés à la mise bas en cas de la fièvre vitulaire ; chez les jeunes, ces carences entrainent un retard de croissance et un faible développement osseux (rachitisme). Ainsi, en comparant ces formes pathologiques aux investigations de terrain de la présente étude, la maladie du Krafft chez le dromadaire ressemble cliniquement à l'ostéoporose dont le déterminisme étiologique reste à explorer chez les animaux examinés par des analyses de laboratoire.

\section{CONCLUSION}

Le Krafft est une maladie chronique qui a affecté les dromadaires pâturant les parcours de Dhahar pendant les saisons hivernale et printanière. La présente enquête épidémiologique, réalisée sur les principaux parcours du Sud tunisien, a révélé que la prévalence de la maladie était très variable entre les troupeaux et entre les régions. Cette maladie a eu diverses causes favorisantes, comme l'augmentation des besoins énergétiques et en minéraux, des climats trop froids et trop secs qui aggravent les lésions articulaires, un surpâturage des autres parcours (El Ouara Tataouine, Jeffara), obligeant les éleveurs à pratiquer la transhumance vers le parcours de Dhahar dont les caractéristiques favorisent l'apparition de la maladie.

D'autres travaux devraient être réalisés pour permettre de mieux maîtriser cette maladie, notamment pour déterminer précisément les causes de l'apparition de la maladie par une meilleure caractérisation des espèces végétales (composition minérale...) ingérées par les dromadaires au cours de leur séjour sur les parcours de Dhahar.

\section{Remerciements}

Les auteurs remercient chaleureusement Bernard Faye et François Meschy pour avoir accepté de donner leurs avis sur ce travail.

\section{BIBLIOGRAPHIE}

1. ABU DAMIR H., 1998. Mineral deficiencies, toxicities and imbalances in the camel (Camelus dromedarius): a review. Vet. Bull., 6: 1110-1119.

2. BLAJAN L., LASNAMI K., 1998. Nutrition et pathologie du dromadaire. Options méditerr. Ser. Semin. : 131-139.

3. DIRECTION GENERALE DE L'ENVIRONNEMENT ET DE LA QUALITE DE LA VIE, 2006. Plan d'action régional de lutte contre la désertification pour le gouvernorat de Médenine. Tunis, Tunisie, ministère de I'Environnement et du Développement durable, 206 p.

4. DURAND M., CHKOUK M., 1958. Le Krafft, une ostéopathie dystrophique du dromadaire. Arch. Instit. Pasteur. Tunis, 35 : 107-152.

5. ESCADAFAL R., 1987. Carte des ressources en sols de la Tunisie, feuille de Tataouine E-S 222. Tunis, Tunisie, ministère de I'Agriculture, direction des Sols, 40 p.

6. FAYE B., BENGOUMI M., 2000. Le dromadaire face à la sous-nutrition minérale, un aspect méconnu de son adaptabilité aux conditions désertiques. Sécheresse, $11: 155-161$.

7. LEVEQUE H., 1957. Contribution à l'étude d'une maladie appelée "Kraff» chez les chameaux du Sud tunisien. Revue Corps vét. Armée, $12: 115-120$.

8. ZAHZAH K., 1981. Etude de la pathologie du chameau du Sud tunisien, le krafft. Thèse Doct. Méd. vét., Ecole nationale de médecine vétérinaire, Sidi Thabet, Tunisie.

9. ZONGPING L., 2004. Studies on rickets and osteomalacia in bactrian camels (Camelus bactrianus). Vet. J.

Accepté le 06.04.2011 


\section{Summary}

Seddik Mabrouk M., Khorchani T., Benromdhane M. Epidemioclinical bases of Krafft disease in camels (Camelus dromedarius) in Southern Tunisia

This study presents a descriptive, analytical, epidemiological, and clinical observation of Krafft disease, as well as an assessment of disease appearance risks by researching receptivity factors, in the southeastern regions of Tunisia. Krafft symptoms are characterized by musculoskeletal disorders leading from lameness to immobilization, which aggravates the condition of the affected animal. Average disease prevalence was $5 \%$ in the herds studied. It has been linked to the passage of herds to Dhahar rangelands. It was mainly observed during winter and spring in females in late gestation and early lactation.

Keywords: Camelus dromedarius - Pasture - Deficiency disease - Mineral deficiency - Tunisia.

\section{Resumen}

Seddik Mabrouk M., Khorchani T., Benromdhane M. Bases epidemio-clínicas de la enfermedad de Krafft en el dromedario (Camelus dromedarius) en el Sur tunecino

Este estudio presenta una observación descriptiva, analítica, epidemiológica y clínica de la enfermedad de Krafft, así como una evaluación de los riesgos de aparición de la enfermedad, mediante la búsqueda de factores de receptividad, en las regiones del sur de Túnez. Los síntomas de Krafft se caracterizan por problemas locomotores, desde cojera hasta la inmovilización que agrava el estado general del animal afectado. La prevalencia media de la enfermedad fue de $5 \%$ en los hatos estudiados. Esta estuvo ligada al paso de hatos hacia los senderos del Dhahar. Esta se observó principalmente durante el invierno y la primavera en las hembras en fin de preñez y al inicio de la lactación.

Palabras clave: Camelus dromedarius - Pastizal - Enfermedad de carencia - Dificiencia de minerales - Túnez. 